\section{Gewichtskontrolle senkt Risiko für Sectio}

\author{
Programme zur Gewichtskontrolle von Schwangeren, die auf Diät und \\ körperlicher Bewegung beruhen, sind unabhängig vom Body-Mass-Index \\ der Frauen erfolgreich. Sie helfen zudem, die Rate an Geburten durch \\ Kaiserschnitt zu senken.
}

— orscher der International Weight Ma- nagement in Pregnancy (i-WIP) Collaborative Group haben die Ergebnisse einer großen Metaanalyse von 36 randomisierten Studien mit 12.526 Schwangeren vorgelegt, in denen es um Wirksamkeit und Nutzen von Diät- und Bewegungsprogrammen zur Gewichtskontrolle während der Schwangerschaft gegangen war. Dabei zeigte sich in den Interventionsgruppen durchweg eine geringere Gewichtszunahme über den Schwangerschaftsverlauf. Im Mittel betrug die Einsparung 0,7 kg (95\%-Konfidenzintervall [ $95 \%-\mathrm{KI}]-0,92$ bis $-0,48$ $\mathrm{kg}$ ). Es spielte keine Rolle, ob die Frauen von Hause aus normal-, übergewichtig oder adipös waren.
Eine andere Frage war, welcher Nutzen sich daraus mit Blick auf bestimmte Schwangerschaftskomplikationen ergab. Im kombinierten Endpunkt aus Gestationsdiabetes, hypertensiven Störungen, Frühgeburten und Kaiserschnitten war kein Unterschied zu den Kontrollprobandinnen feststellbar. Das Risiko, per Sectio zu entbinden, war in den Interventionsgruppen jedoch geringer (Odds Ratio [OR] 0,91; $95 \%$-KI 0,83-0,99).

Eine strengere Trennung zwischen primär bewegungs- und diätbasierten Interventionen förderte weitere Unterschiede zutage. Die bewegungsbasierten Programme senkten auch das Risiko, einen Schwangerschaftsdiabetes zu entwickeln. Diätbasierte Programme hatten
Vorteile bei der Frühgeburtlichkeit; allerdings handelte es sich hier um relativ kleine Studien und das Konfidenzintervall war breit.

Keine Hinweise gab es darauf, dass die geringere Gewichtszunahme der Mutter dem Nachwuchs zugutekäme. Totgeburten, kleine oder große Kinder für das Reifealter und Einweisungen auf die Intensivstation für Neugeborene kamen in Interventions- und Kontrollgruppen gleich häufig vor.

Das Fazit der i-WIP-Gruppe lautet daher: „Auf Diät und körperlicher Aktivität beruhende Interventionen während der Schwangerschaft mindern den Gewichtszuwachs und senken das Risiko für Kaiserschnitte.“

Dr. Robert Bublak

The International Weight Management in Pregnancy (i-WIP) Collaborative Group. Effect of diet and physical activity based interventions in pregnancy on gestational weight gain and pregnancy outcomes: meta-analysis of individual participant data from randomised trials. BMJ 2017; 358: j3119

\title{
ASS als Prophylaxe der frühen Präeklampsie
}

\section{Bei Schwangeren mit hohem Präeklampsierisiko tritt eine frühe Gestose offenbar signifikant seltener auf, wenn sie von der 11.-14. Schwangerschafts- woche an täglich $150 \mathrm{mg}$ ASS einnehmen, wie eine britische Studie zeigt.}

$\mathrm{E}_{\mathrm{B}}^{\mathrm{n}}$ mpfehlungen zur prophylaktischen Einnahme niedrig dosierter Acetylsalicylsäure (ASS) bei erhöhtem Präeklampsierisiko existieren außer in den USA und Großbritannien mittlerweile auch für Deutschland. Über die richtige Patientinnenauswahl und optimale Dosierung ist man sich international jedoch noch nicht einig.

In einer randomisierten, doppelblinden, placebokontrollierten Studie haben Wissenschaftler in London den präventiven Effekt von ASS auf das Präeklampsierisiko von Frauen mit Einlingsschwangerschaften erneut untersucht. $11 \%$ der 26.941 Frauen, die zwischen der 11. und 13. Schwangerschaftswoche (SSW) gescreent worden waren, wurden als Hochrisikopatientinnen für eine frühe Präeklampsie bei einer Gestationsdauer von $<37$ Wochen („preterm pree- clampsia") eingestuft. Die Risikobewertung erfolgte mithilfe eines Algorithmus, der unter anderem den mittleren arteriellen Blutdruck, den Pulsalitätsindex uteriner Arterien, das schwangerschaftsassoziierte Plasmaprotein A (PAPP-A) im Serum sowie den PlacentaWachstumsfaktor (PLGF) berücksichtigte.

In 13 Geburtskliniken in UK, Spanien, Italien, Belgien, Griechenland und Israel wurden 1.776 Teilnehmerinnen randomisiert. Frauen der ASS-Gruppe nahmen von der 11.-14. bis zur 36. SSW täglich 150 mg ASS ein, die Frauen der Kontrollgruppe erhielten Placebo. Das mittlere Alter der Teilnehmerinnen lag bei 31,5 bzw. 31,4 Jahren.

Während es in der ASS-Gruppe bei $1,6 \%$ der Schwangeren zu einer frühen Präeklampsie kam, lag der Anteil unter
Placebo bei 4,3\%. Dies entsprach nach Abgleich anderer Einflussfaktoren einer Risikoreduktion von $62 \%$. Hinsichtlich unerwünschter Ereignisse bei Müttern und Kindern zeigten sich zwischen ASS und Placebogruppe keine signifikanten Unterschiede.

Auch in der aktuellen deutschen Leitlinie „Diagnostik und Therapie hypertensiver Schwangerschaftserkrankungen“ (AWMF-Register Nr. 015/018) steht, dass die „derzeit einzige effektive Prävention der Präeklampsie bei Frauen mit Risikofaktoren (z.B. schwere Präeklampsie in der Anamnese) in einer ab der Frühschwangerschaft (bis spätestens 16. SSW) beginnenden oralen Einnahme von niedrig dosierter ASS (75$150 \mathrm{mg} / \mathrm{Tag}$ )“ besteht. Den Leitlinienautoren zufolge hat sich hierzulande eine Dosierung von $100 \mathrm{mg} / \mathrm{Tag}$ bis zur 34 . SSW etabliert. Eine generelle ASS-Prophylaxe sei nicht indiziert.

Dr. Christine Starostzik

Rolnik DL et al. Aspirin versus Placebo in Pregnancies at High Risk for Preterm Preeclampsia. NEJM 2017, online 28. Juni;

https://doi.org/10.1056/NEJMoa1704559 